\title{
An investigation on skeleton-based top-down modelling approaches of complex industrial product
}

\begin{abstract}
In industry, today's approach to assembly design is still largely based on a bottom-up approach which, in contrast with the most advanced top-down techniques, is unfit to deal with very large and complex products. The reason for this lies in the high number of relationships to be established between parts and in the lack of a high-level control of the assembly design. This makes the management of design changes a labor-intensive process and the capture of design intent difficult to achieve. The paper, referring to the most advanced research fields of Concurrent Engineering and Knowledge-Based Engineering, focuses on a top-down modelling approach based on skeleton, which constitutes the most natural but still scarcely exploited way to attain a high reactivity to design modifications. Through the application of suitable methodologies, such as that one for a SKeLeton geometry-based Assembly Context Definition (SKL-ACD), the skeleton is also able to capture and codify assembly process engineering information since the early phases of the product development process. With the purpose of promoting the knowledge of these skeleton-based modelling techniques, that have a great relevance for training professional, technical and mechanical engineers, this paper implements the SKL$A C D$ methodology to an industrial case study in order to identify, with a unique and repeatable workflow, the reference geometrical entities and the mutual relationships to embed into the product skeleton. The skeleton types and the related fields of use are also described, placing particular emphasis on problems or shortcomings still not resolved, especially in consideration of the need to assist the designer in defining the impact of a parameter on assembly modification and in avoiding loops while defining formulas. A new tool, in the form of a multilayer graph, is finally proposed that is able to display and differentiate clearly the formulas, the design parameters and the impact of their modification on skeleton entities and members of the assembly.
\end{abstract}

\section{KEY WORDS}

Knowledge-Based Engineering, Top-Down Assembly Design, Skeleton, Product Parametrization

\section{Introduction}

The design process today is influenced by the need for better, less expensive, and faster-to-market products. This requires the implementation of innovative strategies capable of achieving and maintaining a consistently

\section{Claudio Ciaccioli Anna Eva Morabito}

University of Salento, Department of Engineering for Innovation, Lecce, Italy

Corresponding author: Anna Eva Morabito e-mail:annaeva.morabito@unisalento.it

First received: 8.7.2020. Accepted: 7.9.2020. 
basic premises for Concurrent Engineering (CE), i.e. a workflow where the phases of the product development process are no longer in rigid succession with each other, but run simultaneously. Thanks to this strategy, the early design process does not focus only on the basic functional aspects of the product but also on specific issues of downstream stages, such as design analysis, manufacturing simulation, assembly sequence planning, production, maintenance, disposal, etc. This allows concentrating the most of design modifications in the early phases of the development process, when they are cheaper and the product design is more flexible.

The traditional design approach is based on parametric and feature-based CAD models, which give a 3D digital representation of the product geometry enriched with technical and technological information and capable of capturing, if well parameterized, the design intent. These CAD models, however, cannot describe the design choices that led to a specific product configuration. Knowledge Based Engineering (KBE) is the design methodology that, by employing specific software tools, is capable to enrich the CAD model by embedding design rules and product and process engineering principles (for example manufacturing data, tooling data and structural information) in order to formalize the design rationale. In doing so, KBE allows capturing and systematically reusing product and process engineering knowledge. This is crucial for reducing design time and costs, automating repetitive operations and being supportive for conceptual design (Rocca, 2012; Chandrasegaran et al., 2013). The knowledge capture or acquisition is a critical phase for KBE diffusion, especially when dealing with conceptual design problems. These problems are usually wicked or ill defined (i.e. incomplete and contradictory) and with changing requirements that are often difficult to recognize. FBS (Function-Behavior-Structure) Ontology is the most promising and widespread solving approach, which categorizes the properties of a product to design or to innovate into three levels: function ("what the object is for"), behavior ("what the object does") and structure ("what the object consists of") (Gero \& Kannengiesser, 2014).

Parallel execution of tasks and cooperation between teams also require the adoption of a top-down product design approach, which begins by defining the product structure before detailing its individual parts (Vielhaber et al., 2004). Thanks to this approach, a product vision, which can be enriched with engineering knowledge, is available from the earliest stages of the development process.

Observing from a more closely CAD perspective, the top-down design paradigm can be very efficiently implemented by a skeleton-based assembly modeling approach. The skeleton is a control structure containing the reference geometry that will drive the key dimensions and positions of the components of the assembly, together with the relative space allocations. This allows efficiently managing the scalability and responsiveness of the product to the propagation of changes made in accordance with the design intent.

The skeleton is also potentially able to represent the knowledge of the product coming from downstream stages of the development process. Recently, various methodologies have been proposed that, starting from data on assembly sequence planning or manufacturing process, compute and define automatically the geometry of product skeleton. Among these, the methodology for Skeleton geometry-based Assembly Context Definition (SKL-ACD) aims at integrating assembly process engineering knowledge in the early phases of the product development process (Demoly et al., 2011). This methodology, which differs significantly from the traditional one where the assembly sequence is defined after the detailed design phase, improves the productivity and efficiency of the design by reducing the iterations due to the definition of poor assembly requirements. A CAD model of the industrial product, suitably parameterized, knowledge-based and capable of acting as master model for a family of products can be, therefore, obtained reducing both repetitive tasks for the designer and time to market for the company.

In order to promote the use of skeleton-based modelling techniques able to integrate the knowledge coming from the downstream phases of the development process, this paper first describes the main steps of the SKL-ACD methodology through the implementation of a simple case study that allows students to understand the design issues involved. The application of SKL-ACD methodology allows identifying the reference geometry to be incorporated into the skeleton. Current CAD systems offer different types of skeleton. This paper describes them by underlining the related fields of use and placing particular emphasis on open issues or shortcomings still not resolved, especially in consideration of the need to assist the designer in defining the impact of a parameter on product design and in avoiding loops while defining formulas.

Particularly, a problem still addressed unsatisfactorily by current CAD systems, concerns the availability of tools capable of visualizing and managing efficiently the product parametrization so that design modifications propagate in agreement with the design intent. The designer, in fact, chooses a set of parameters to guide the skeleton and quickly change some key variables (such as dimensions, positions and space allocations) of the assembly members. Since these modifications are propagated, through the skeleton, to the parts or sub-assemblies downstream, the designer should be always aware of which members are affected by a specific parameter. In order to navigate this domain of relations, CAD software currently implement a set 
of tools, which, as will be shown by this paper, are not always able to support adequately the designer activities. After having given an overview of these tools, the paper proposes the construction of a multilayer graph, as a new way to visualize and manage effectively the paths through which the parameters of the skeleton influence the product geometry. In the case of a top-down design approach, such as the skeleton-based one for which the reuse of parameters makes the tracing of the related paths more complex, this issue is actually more felt.

The paper has the following outline. Section 2 surveys, from a CAD perspective, the concept of product skeleton pointing out the basic principles for a topdown modelling approach based on skeleton. Section 3 describes synthetically the case study for which the steps of the SKL-ACD methodology, used to identify the skeleton's reference geometry, are then explained. Section 4 overviews the main skeleton types and the related fields of use. Particular emphasis is placed on the tools currently implemented to visualize and manage the paths of skeleton parameters and formulas. Various aspects of the issues addressed are highlighted and discussed in section 5, where a multilayer graph is proposed as a new visualization tool to better support the designer when tracking parameters paths. Finally, conclusions are provided in section 6 .

\section{Skeleton-based modelling approach}

The implementation of a collaborative and simultaneous design approach leads, from a CAD perspective, to the modification of the assembly design paradigm from bottom-up to top-down (Vielhaber et al., 2004). In conventional top-down approach, the parts are modelled in the context of the assembly referencing the geometry of existing components, i.e. creating external references pointing to existing geometry in the assembly. Any geometrical element (sketch, point, line, curve, surface, etc.) defined in a component can be reused to model and to control another part, referred to as contextual part. When designing with this approach (often also referred to as "design in context"), the contextual part is automatically updated as soon as the geometry of the referenced component changes. A contextual link between the driving (or referenced) component and the driven (or contextual) component is then established.

Figure 1(a) simply illustrates this approach through the example of a product made of three blocks, shown in exploded view for clarity. The yellow and orange parts (respectively called Part 2 and Part 3 in the graph reported on the right side of Figure 1(a)) have been modelled in the context of the assembly from the sketches obtained by projecting respectively the two edge loops delimiting the Part 1's pockets. An equivalent result can be obtained by copying associatively the sketches of these pockets respectively into Part 2 and Part 3. In doing so, contextual links are established between Part 1 and Part 2, and Part 1 and Part 3 (as shown by arcs of the graph reported on the right side of Figure 1(a)). Therefore, any change on pockets' sketches of Part 1 is automatically propagated to the other parts.

In the conventional top-down approach, additionally, a component's parameter can be reused in another part to connect the related geometries. This occurs through the creation of relations (i.e. formulas), by which any change made to the referenced parameter will be reflected in the others by modifying the related geometries.

The skeleton-based approach identifies a specific way to define relationships between parts by centralizing them on the skeleton itself. Figure 1(b) shows the implementation of this approach to the plain example of the three blocks. The skeleton is composed of three sketches and each of them is either directly referenced during the geometric modelling of the related part or associatively copied inside this one. In this last case, an external reference is created within each part well before detailing the corresponding geometric model. Consistently, the arcs of the graph of Figure 1(b) represent the contextual links between skeleton and parts of the assembly. Any change in the skeleton' sketches will propagate to the other contextual parts by modifying the related geometries.
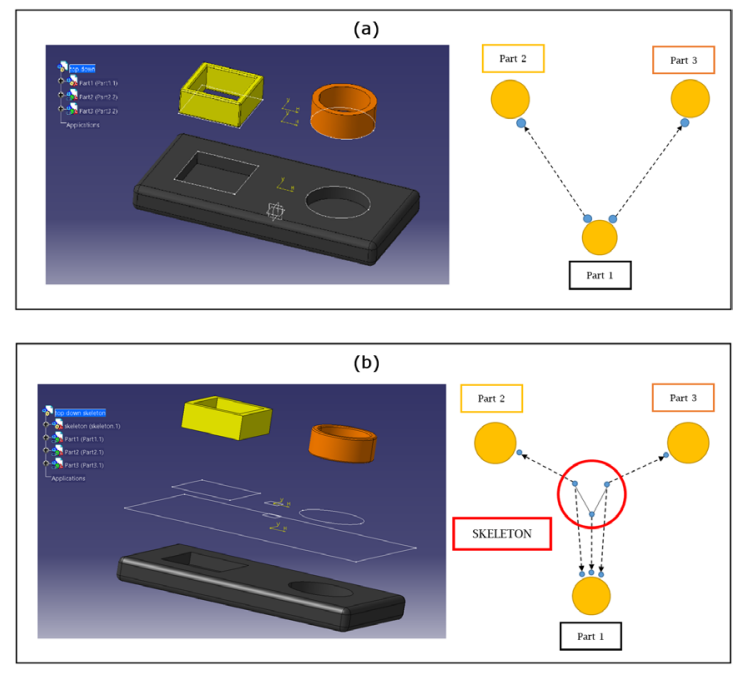

» Figure 1: Top-down modelling approaches: conventional (a) and with skeleton (b)

Skeleton geometry can also be used to position the components of the product through the selection of a suitable set of assembly constraints that establish positional links between the skeleton and the components of the product. In order to capture the design intent, skeleton parameters can be created and then referenced by the assembly members 
through the definition of formulas. The assembly members reuse, therefore, the skeleton's elements to define the related underlying design framework.

Any change to the overall design can be made on the skeleton model and, by means of the before-mentioned relationships (i.e. contextual, positional and by formulas), is automatically propagated to the driven components. This allows an improved management of high-level design modifications. As the components are not directly linked to each other, the deletion of a component within an assembly will not affect the others. Moreover, since the skeleton model does not use external references within the assembly to define its geometry, the issue related to the circular references is avoided. All external references, in fact, only point to the skeleton model so that the direction of information is always downwards, from the skeleton model to the other components and the relationships cannot interfere with each other. Another advantage of this approach is that the components can be edited separately making tasks parallelization and cooperation possible between different professional figures. Thanks to the afore-mentioned relationships between skeleton and components, in fact, the designer can be sure that design data is up to date.

The skeleton-based approach may be also useful to address issues related to the protection of intellectual property. When designing a product, companies usually make use of third-party components. In these circumstances, the intellectual property of a participating company should be protected from the others. This safeguard should not be an obstacle to product design collaboration. Moreover, each enterprise usually utilizes a different CAD software, which represents a further obstacle for collaboration and designers often rely on ISO 10303 STEP as interchange file format that however is not parametric by nature. Skeleton design can address these issues by becoming a neutral reference model (Mun, Hwang \& Han, 2009): each company involved in product design can work on the reference geometry of the skeleton, which does not contain any detailed description of the parts, but only the information necessary to meet the requirements of the other collaborating companies.

\section{The SKL-ACD methodology}

Industrial plants generally employ heat exchangers of different sizes so that the application of a skeleton-based CAD approach to the design of a pipe bundle cleaner can be beneficial for scalability purposes. A pipe bundle cleaner is an automatic device, designed for cleaning heat exchangers and other kinds of equipment that experience fouling: it consists of three main functional groups, namely a scissor lift, a lance track and a lance slider. The machine operates introducing sequentially into each tube of the exchanger a rigid lance with a high-pressure nozzle threaded on one end.
At the other end, high-pressure water is pumped (up to 3000 bar) with flows that reach hundreds of liters per minute; thus, the hydrodynamic action removes deposited materials that previously choked the tubes.

The tube bundle appears as a matrix of thousands of holes that can reach 2 meters in diameter; the tube length varies upon the size of the heat exchanger, usually from 6 to 11 meters.

In reference to the coordinate system in Figure 2, the scissor lift is responsible for the movement along the Z-axis. The rigid lances lay on the lance track, which can slide along the scissor lift table on the $Y$-axis by a chain transmission. The combination of $Z$ and $Y$ translations guarantees the correct positioning of the lances with respect to the holes matrix. The lance slider, where the hydrodynamic connections with the lances occur, can slide along the $\mathrm{X}$-axis via another chain transmission, introducing the lances into the heat exchanger and beginning the cleaning process.

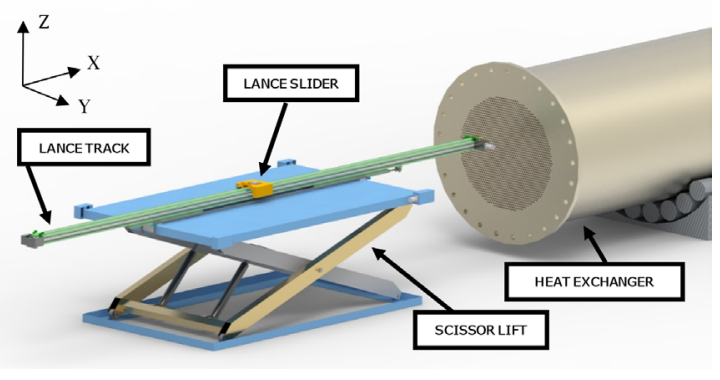

» Figure 2: Rendering of the cleaning process

The SKL-ACD methodology allows defining the skeleton's reference geometry consistently with the product structure and the assembly sequence planning engineering information (Demoly et al., 2011). The product structure is identified starting from existing design concepts or Bill Of Materials (BOM) of similar previous designs retrieved from the company know-how. Table 1 shows the 15 parts or groups of parts which make up the product structure for the case study considered.

Starting from the assembly sequence of the product, the part-to-part relational information on physical contacts and precedence constraints are embedded in a directed graph and in the related adjacency matrix. Each vertex of the directed graph is a part or sub-assembly, while each edge identifies a relationship between vertices. In the directed graph for the pipe bundle cleaner of Figure 3, the vertices are the parts or the groups from Table 1. The precedence constraints are represented by a dotted line and apply to the chain transmissions of both lance track (part 8 of BOM of Table 1) and lance slider (part 13 of Table 1). These transmissions, in fact, need to be mounted after the other components are in place for interference reasons. 
Table 1

BOM for the pipe bundle cleaner

\begin{tabular}{c|c}
\hline Num. & Name \\
\hline 01 & BASE \\
\hline 02 & H_FRAME \\
\hline 03 & U_FRAME \\
\hline 04 & PIN \\
\hline 05 & HYC_Z \\
\hline 06 & TOP \\
\hline 07 & BRACKETS \\
\hline 08 & LANCE_TRACK \\
\hline 09 & LOCK_FRAME \\
\hline 10 & YMT \\
\hline 11 & YIT \\
\hline 12 & HYC_X \\
\hline 13 & LANCE_SLIDER \\
\hline 14 & XMT \\
\hline 15 & XIT \\
\hline
\end{tabular}

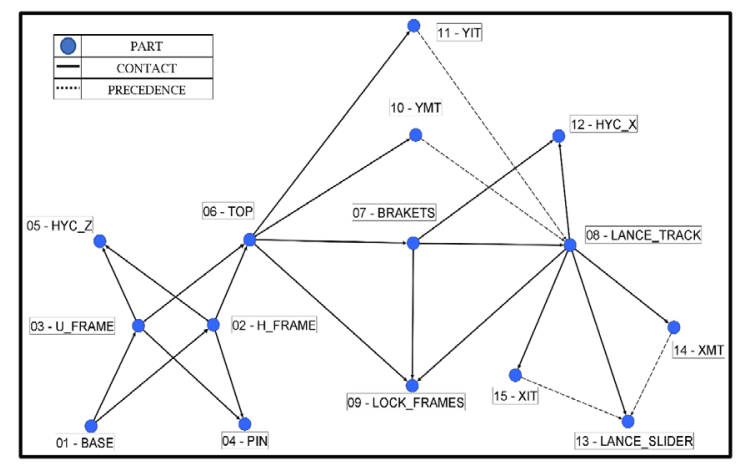

» Figure 3: Directed graph for the pipe bundle cleaner

Figure 4 represents the adjacency matrix for the case study.

\begin{tabular}{c|c|c|c|c|c|c|c|c|c|c|c|c|c|c|c}
\hline & $\mathbf{0 1}$ & $\mathbf{0 2}$ & $\mathbf{0 3}$ & $\mathbf{0 4}$ & $\mathbf{0 5}$ & $\mathbf{0 6}$ & $\mathbf{0 7}$ & $\mathbf{0 8}$ & $\mathbf{0 9}$ & $\mathbf{1 0}$ & $\mathbf{1 1}$ & $\mathbf{1 2}$ & $\mathbf{1 3}$ & $\mathbf{1 4}$ & $\mathbf{1 5}$ \\
\hline 01 & 0 & 1 & 1 & 0 & 0 & 0 & 0 & 0 & 0 & 0 & 0 & 0 & 0 & 0 & 0 \\
\hline 02 & -1 & 0 & 0 & 1 & 1 & 1 & 0 & 0 & 0 & 0 & 0 & 0 & 0 & 0 & 0 \\
\hline 03 & -1 & 0 & 0 & 1 & 1 & 1 & 0 & 0 & 0 & 0 & 0 & 0 & 0 & 0 & 0 \\
\hline 04 & 0 & -1 & -1 & 0 & 0 & 0 & 0 & 0 & 0 & 0 & 0 & 0 & 0 & 0 & 0 \\
\hline 05 & 0 & -1 & -1 & 0 & 0 & 0 & 0 & 0 & 0 & 0 & 0 & 0 & 0 & 0 & 0 \\
\hline 06 & 0 & -1 & -1 & 0 & 0 & 0 & 1 & 0 & 1 & 1 & 1 & 0 & 0 & 0 & 0 \\
\hline 07 & 0 & 0 & 0 & 0 & 0 & -1 & 0 & 1 & 1 & 0 & 0 & 1 & 0 & 0 & 0 \\
\hline 08 & 0 & 0 & 0 & 0 & 0 & 0 & -1 & 0 & 1 & $-\lambda$ & $-\lambda$ & 1 & 1 & 1 & 1 \\
\hline 09 & 0 & 0 & 0 & 0 & 0 & -1 & -1 & -1 & 0 & 0 & 0 & 0 & 0 & 0 & 0 \\
\hline 10 & 0 & 0 & 0 & 0 & 0 & -1 & 0 & $\lambda$ & 0 & 0 & 0 & 0 & 0 & 0 & 0 \\
\hline 11 & 0 & 0 & 0 & 0 & 0 & -1 & 0 & $\lambda$ & 0 & 0 & 0 & 0 & 0 & 0 & 0 \\
\hline 12 & 0 & 0 & 0 & 0 & 0 & 0 & -1 & -1 & 0 & 0 & 0 & 0 & 0 & 0 & 0 \\
\hline 13 & 0 & 0 & 0 & 0 & 0 & 0 & 0 & -1 & 0 & 0 & 0 & 0 & 0 & $-\lambda$ & $-\lambda$ \\
\hline 14 & 0 & 0 & 0 & 0 & 0 & 0 & 0 & -1 & 0 & 0 & 0 & 0 & $\lambda$ & 0 & 0 \\
\hline 15 & 0 & 0 & 0 & 0 & 0 & 0 & 0 & -1 & 0 & 0 & 0 & 0 & $\lambda$ & 0 & 0 \\
\hline
\end{tabular}

» Figure 4: Adjacency matrix for the pipe bundle cleaner

The numbers populating this matrix have a sign that reflects the directionality of the part-to-part relation, namely the verse of the arrow for the generic edge of the directed graph. The element $a_{i j}$ of this matrix is +1 or $+\lambda$ if it represents respectively a contact or precedence relation that starts from the part associated to the $i^{\text {th }}$-row and leads to the part corresponding to the $\mathrm{j}^{\text {th }}$-column. Conversely, it is -1 or $-\lambda$ if describes respectively a relation starting from the part of the $j^{\text {th }}$-column and leading to the part of the $\mathrm{i}^{\text {th }}$-row.

Each edge of the directed graph also identifies a potential kinematic/technological pair (or joint), which can be of different kind based on the relative degrees of freedom (DOF) allowed by the part-to part relative motion. Assembly constraints and skeleton geometric entities can be generated, therefore, for each type of joint by means of an appropriate codification (e.g. a revolute joint is defined by the pairs line-line and plane-plane). Figure 5 shows the results of this step for the pipe bundle cleaner.

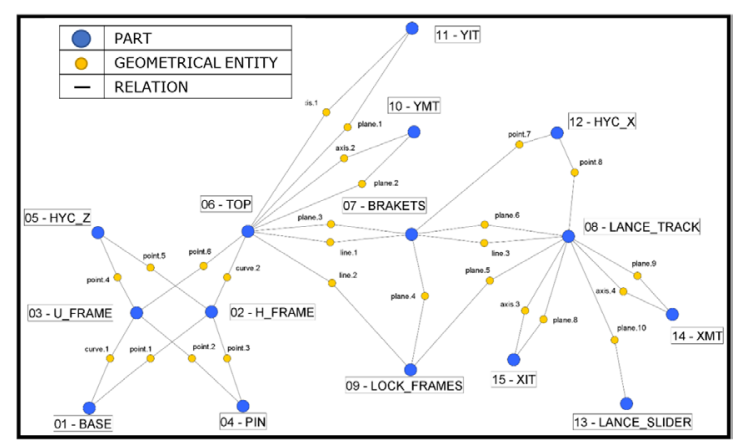

» Figure 5: Skeleton entities definition

Based on the 13 constraints described in the Technologically and Topologically Related Surfaces (TTRS) theory (Desrochers \& Clément, 1994), new constraints between the skeleton entities can be then defined. A new graph, called "skeleton graph", is therefore built based on these geometric entities and mutual relationships. Figure 6 shows the "skeleton graph" for the pipe bundle cleaner.

The geometric model of the skeleton emerges from this graph through the CAD modelling of the afore-mentioned geometric entities and of the mutual geometric relationships. Consistently with these data, the skeleton incorporates also a set of parameters so that the CAD model of the pipe bundle cleaner is able of acting as master model for a whole family of products, reducing both repetitive tasks for the designer and time to market for the company.

Table 2 shows the 15 skeleton parameters able to drive the pipe bundle cleaner geometry and the relative variability ranges. Among these, 11 parameters (in red) have been identified in order to drive the variability of product dimensions. The other 4 (in green), directly related to geometrical relations between the skeleton entities, are kinematic parameters representing the actual degrees of freedom (DOF) of the machine. 


\section{CAD techniques for skeleton- based modelling approach}

Three are the main modelling techniques aimed at generating as many skeleton typologies; they are named respectively:

- Static skeleton

- Kinematic skeleton

- Knowledge-driven skeleton

This paper focuses on the first two types, which have an immediate application on the designer's activities, although they are still underutilized particularly by small and medium-sized companies. The techniques for modelling knowledge-driven skeletons, on the other hand, need to be contextualized in a company framework for the necessary knowledge acquisition and require object-oriented programming to formalize know-how.

The static skeleton is described by a single part able to drive the assembly geometry and it is useful to the topdown design of assemblies for which one does not deem necessary to perform a detailed kinematic analysis.

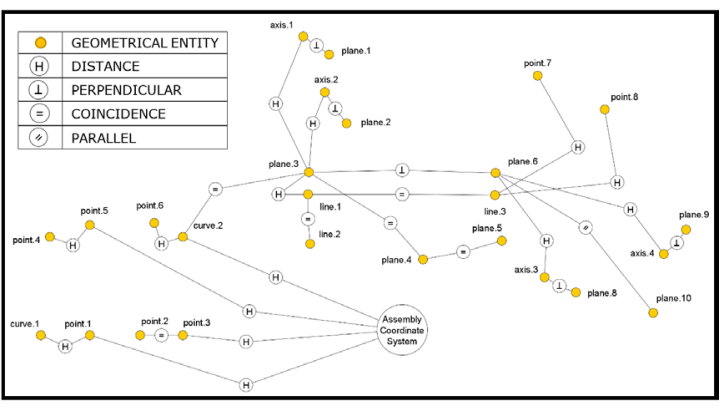

Figure 6: Skeleton graph
Referring to the case study under examination, Figure 7(a) shows the static skeleton modelled within CATIA V5. This is constructed as a part containing the reference geometrical entities and the mutual relationships shown in the graph of Figure 6. During the modelling of a member of the assembly, these entities are referenced by direct selection or copied associatively. Figure 7(a) also illustrates in red the reference geometrical entities required by the TOP (i.e. part 6 of the BOM in table 1), which is shown in Figure $7(b)$.

a)

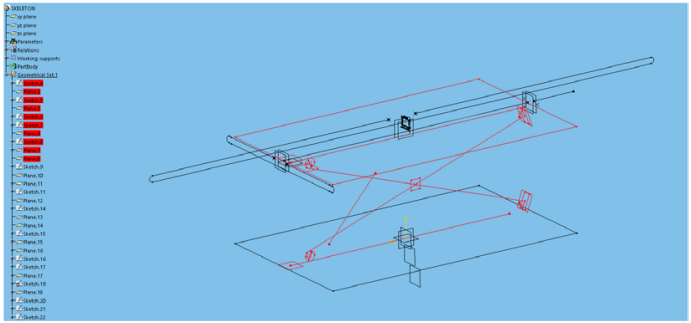

b)

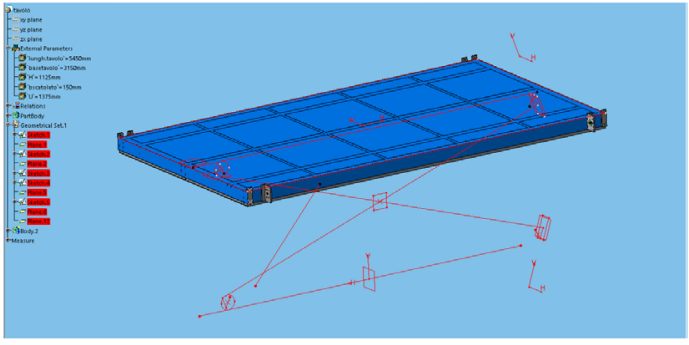

» Figure 7: Static Skeleton modelled in CATIA V5 for the case study (a). The TOP part of the case study with the skeleton entities "pasted with link"

This way of "fixing" each part to a common static frame (i.e. the static skeleton) allows investigating only instantaneous product configurations. In other terms, by varying the kinematic parameters of the static skeleton in the constraints respect (such as those of the sketch

Table 2

Skeleton parameters for the pipe bundle cleaner

\begin{tabular}{|c|c|c|c|}
\hline Num. & Code & Range/Formula [mm] & Description \\
\hline 01 & B & $3000-5000$ & Scissor length \\
\hline 02 & BS & $100-150$ & Hollow section base \\
\hline 03 & $\mathrm{HS}$ & $200-300$ & Hollow section height \\
\hline 04 & $\mathrm{H}$ & $\mathrm{BT} / 4$ & $\mathrm{H}$ frame span \\
\hline 05 & U & $\mathrm{H}+250$ & U frame span \\
\hline 06 & $\mathrm{SH}$ & $150-200$ & Square hollow section \\
\hline 07 & $\mathrm{HT}$ & $600-3600$ & Table height (z) \\
\hline 08 & BT & $3150-3500$ & Table base \\
\hline 09 & LT & $B+300$ & Table length \\
\hline 10 & TY & $-1300-1300$ & Lance track translation (y) \\
\hline 11 & LB & $3650-5650$ & Lance track length \\
\hline 12 & TX & $0-500$ & Lance translation $(\mathrm{x})$ \\
\hline 13 & $\mathrm{TC}$ & $-5650-5650$ & Lance slider translation $(\mathrm{x}$ ) \\
\hline 14 & S & $5-8$ & Hollow section thickness \\
\hline 15 & $\mathrm{C}$ & $\left(0.02805^{*}\right.$ B' $\left.^{\prime} * * 2+0.08045^{* ` B}{ }^{\prime}-0.18\right)$ & Cylinders arm \\
\hline
\end{tabular}


of Figure 8 belonging to the case study's skeleton), it is possible to visualize the kinematic behaviour of the product. This model, however, remains a substantially rigid structure that does not allow to answer any query about the relative motion between the parts (such as to determine the direction of motion of the assembled parts with respect to other parts or to find the velocity and acceleration of any point on any part during the motion of the mechanism).

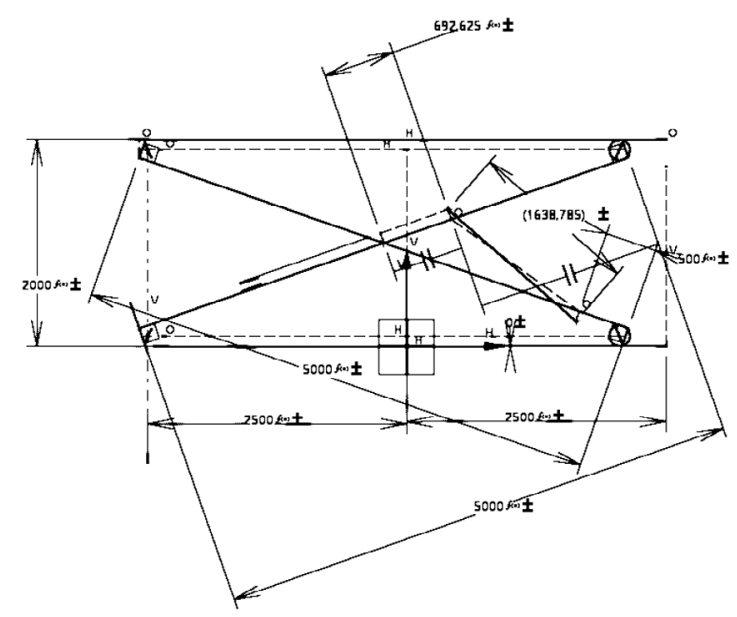

» Figure 8: $2 D$ constraints embedded in a sketch of the static skeleton constructed within CATIA V5 for the case study

The kinematic skeleton is modelled as an assembly consisting of parts, each containing only the geometric entities that will be referenced by the related member of the assembly. Within CATIA, for example, the construction of this type of skeleton takes place with a technique that actually overturns the classic bottom-up use of software modules exemplified by the workflow sequence: Part Design -> Assembly Design -> Digital MockUp (DMU) kinematics. In this case, perfectly in line with a top-down methodology, the user has to switch to the kinematic module to define the joints and the fixed part in order to create a mechanism from the skeleton parts. By so doing, the software automatically defines also the minimal number of necessary assembly constraints. Then, the actual geometry of the parts is defined referencing directly the underlying skeleton geometry. Finally, each part is linked with its own skeletal part through the command called "Mechanism Dress up".

Currently there are CAD systems (e.g. CREO) which have built-in features specifically devoted to skeleton modelling and available since the early steps of the product design. In other cases, as in CATIA V5, the distinction between static and kinematic skeleton is only procedural, so that it may be very difficult to switch typology when the assembly design is in an advanced state. Additionally, there are systems where the distinction between static and kinematic skeleton is useless. SOLID EDGE, for example, contrary to CATIA V5 allows the creation of $2 \mathrm{D}$ reference geometry (sketches, planes, etc.) directly at the product level. In other words, the assembly is not merely a set of parts and relationships, but it may contain its own geometry, which can be selected and referenced by lower level members through a projection operation. Thus, the assembly level constitutes a natural place to define skeletons, parameters and links. Figure 9(a) shows the pipe bundle cleaner assembly, constructed from reference datums and sketches defined at the assembly level. If some reference geometry is projected downstream while designing a part, a "links" node is created into the assembly tree, as shown in Figure 9(b), which constitutes the associative reference set for that specific part. Moreover, a "variables" node is also generated which includes the published parameters in use from the upper assembly level.

As for the workflow within SOLID EDGE, the Motion analysis module (devoted to the kinematic analysis of the mechanism) does not require the implementation of a specific type of skeleton, making the distinction between static and kinematic skeleton useless. Consequently, a skeleton can be designed by using the reference geometry that drives simultaneously several parts or
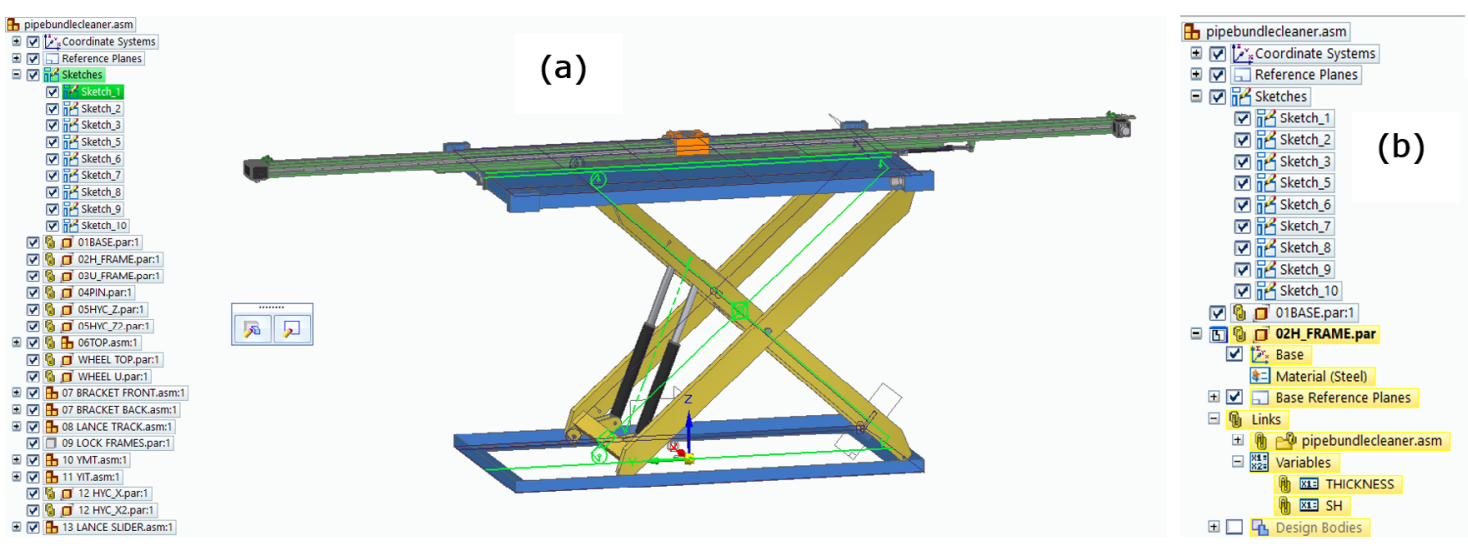

» Figure 9: The pipe bundle cleaner and the related skeleton (highlighted in green) constructed within SOLIDEDGE (a).

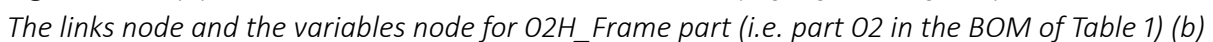




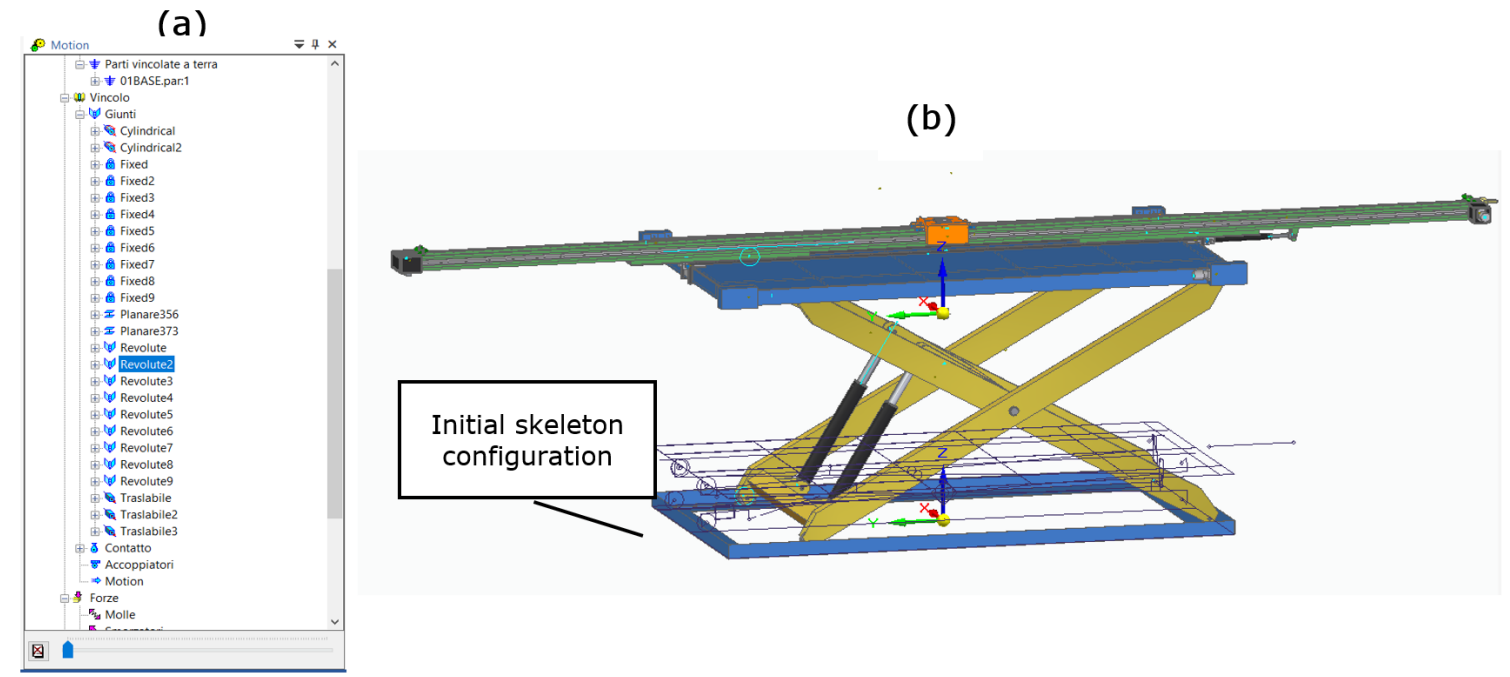

» Figure 10: Motion tree (a) and Motion simulation with skeleton as initial configuration (b)

part-by-part and then stored in a specific sub-assembly. The motion analysis will still act as a different scenario where functional simulation groups can be defined independently of how the product tree is hierarchically organized. Figure 10(a), for example, shows a motion tree for the case study that is different from the product tree. The skeleton can be used as a reference for an initial kinematic configuration but the joints defined in the motion environment are independent from the constraints defined between skeleton and parts. Consequently, while defining motion constraints, e.g. a curve on curve joint, skeleton geometry cannot be used and it is necessary to model the single parts first and then project those 3D features needed for joints definition. In Figure 10(b), the motion analysis of the lifting platform has been carried out, starting from the lower position defined by the initial skeleton configuration.

\section{CAD tools for visualization and management of product parametrization}

The dimensional and kinematic parameters listed in Table 2 are created and embedded into the skeleton geometry through the definition of formulas (or relations). In order to drive the product geometry, linked copies of these parameters are created in the corresponding parts, where they are usually listed under the node of the external parameters. Although only 11 parameters were required to guide the scalability of the case study (i.e. to make the scissor lift and lance track scalable to various sizes of heat exchangers), the number of relations to be defined is rather high. For this reason, it is important that CAD systems include some tools for the tracking of information flows in the parameter network of the assembly. Many software, such as CATIA and SOLID EDGE, use a table or entry list for defining, visualizing and managing formulas and parameters. Figure 11 shows, for example, the table with filters offered by CATIA V5.

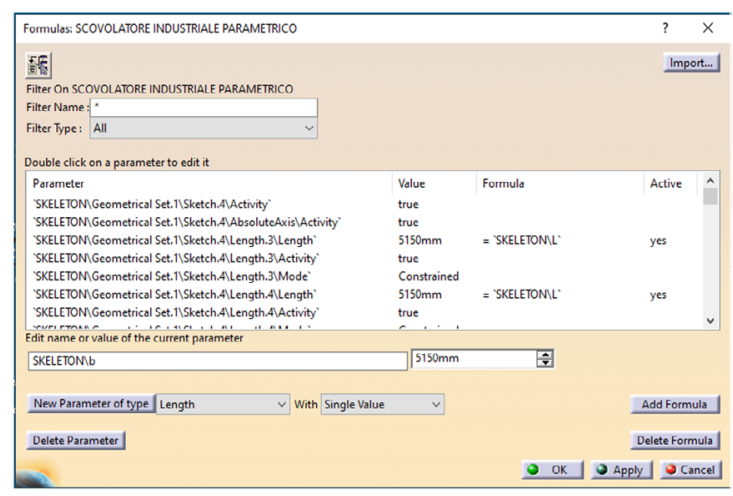

" Figure 11: The table with filters offered by CATIA V5 to describe the formulas

Similarly, the "Peer Variables" command in SOLID EDGE opens an interface (shown in Figure 12) where the relations of every part of the assembly can be inspected and grouped, for example based on the respective sketch where they have been defined. This interface, always organized in the form of a table lets the user define formulas, link parameters downstream, establish value ranges and create simple IF-THEN rules.

To manage and visualize the parameters of a product effectively, it may be advisable to create a GUI interface. Figure 13 shows an example of GUI implemented by a VBA macro in CATIA V5 for the case study under examination. This type of interface is useful for verifying the propagation of the modification and ensuring that the variation of the driving parameters in the relative ranges does not generate impossible geometries and/or interferences between the members of the assembly. Moreover, since a VBA macro runs in background, the product geometry regeneration is faster than a manual update in the CAD environment. 


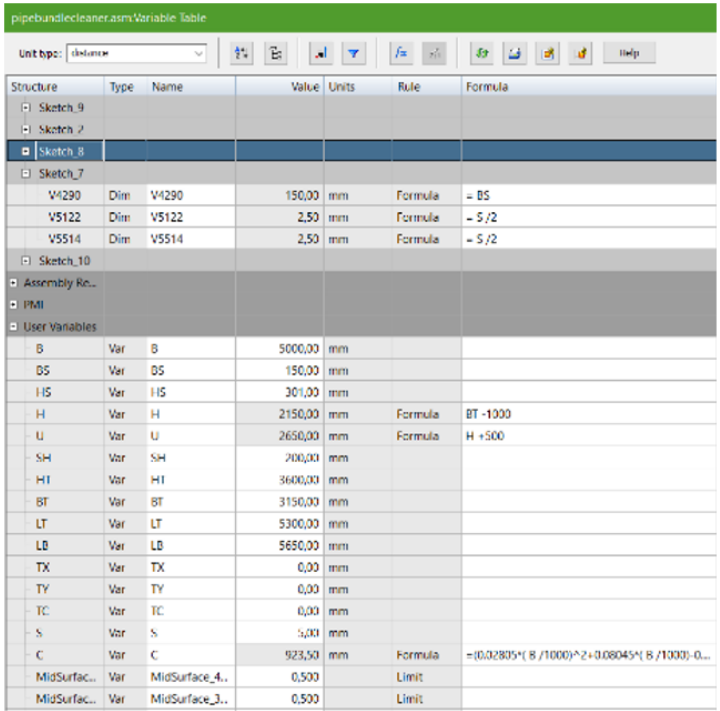

» Figure 12: The table generated by the "Peer Variables" command in SOLID EDGE

For a designer who needs to be supported in defining the impact of a parameter on modifying product geometry and in avoiding loops while defining formulas, the graphbased tools are more effective. Single-layer graphs, also filtered for considering only a certain type of relationships (i.e. positional, contextual and by formulas), can be generated within CAD systems such as CATIA V 5 and CREO. The impact of the single parameter, on the other hand, can be investigated, by querying its parent-child relationships. However, these relationships are always limited to the context of the part under consideration. Not being able to visualize the complete path of the parameter, even if a contextual link exists downstream, is evidently a limit of the current CAD systems especially if one considers that the parameter of a skeleton generally guides the dimensions of several parts of an assembly.

Recently, some researchers have addressed the problems of the documentation and visualization of complex networks of parametric-associative information within 3D CAD models, mostly proposing networks of planar relationships (Marchenko et al., 2011). However, an issue still exists concerning the development of effective visualization tools for supporting the designer in identifying and keeping track of parameters and formulas in a CAD product. This problem, as confirmed also by the investigation carried out here, is evidently even more critical in the case of a topdown design approach where the reuse of parameters makes the tracing of the related paths more complex.

Using the latest and most advanced tools of multilayer network visualization (McGee et al., 2019), such as Trixzpicture of MuxViz, the product links, once extracted from the CAD database with a certain effort, can be rearranged spatially in a more effective way. Figure 14 shows a new type of graph, referred to as Skeleton String Model (SSM), proposed here in order to display and manage more efficiently the $C A D$ assembly parameters and formulas.

The SSM is a multilayer graph organized into two layers: the bottom layer is a graph very similar to that one shown in Figure 5, where the nodes are the parts/components of the product. An arc between two nodes is established when a skeleton entity is referenced by both the members associated with the two nodes respectively. Multiple arcs, therefore, may connect a given couple of nodes. The upper layer is a graph where the nodes represent the skeleton parameters. In the specific case

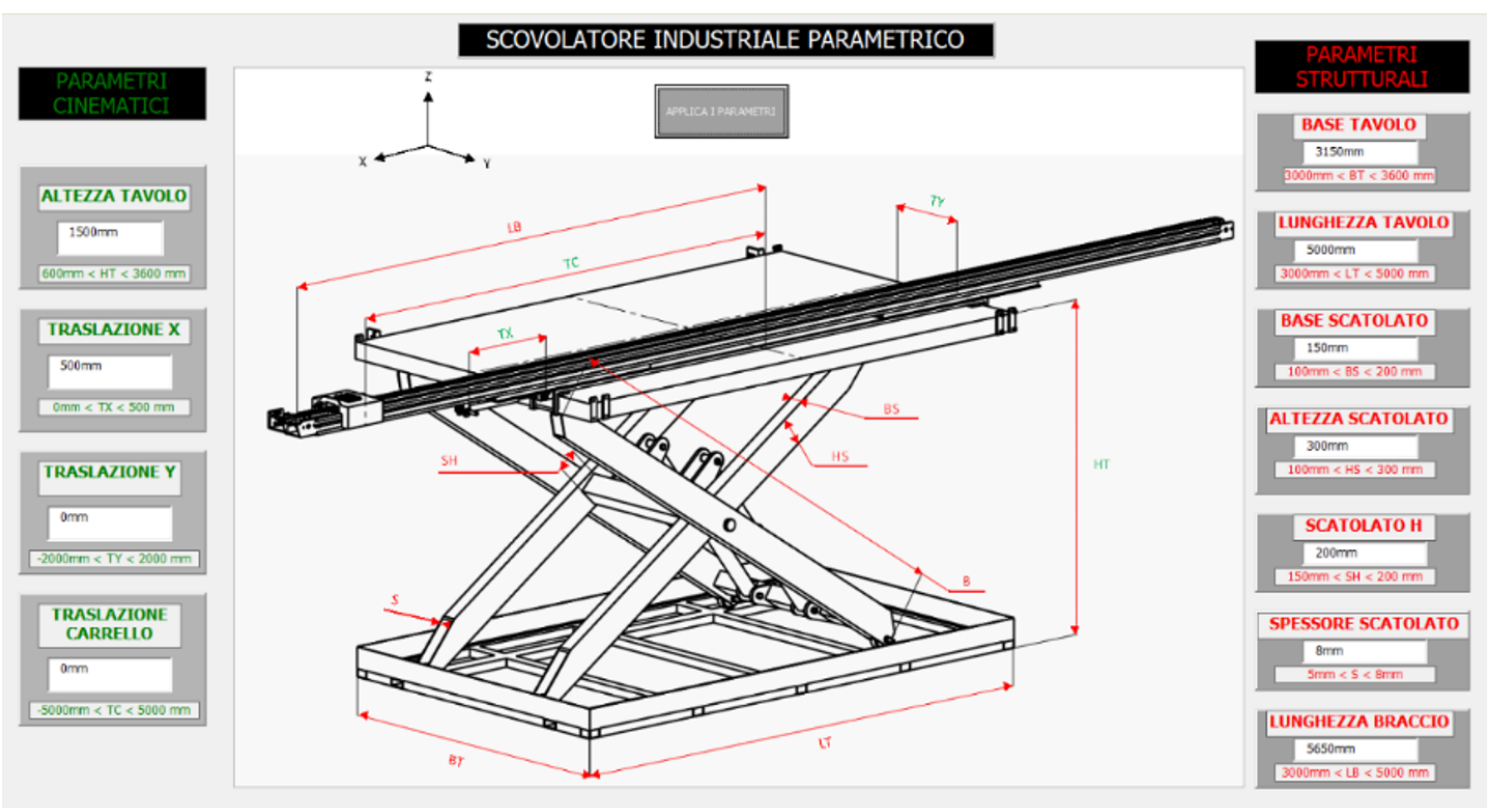

» Figure 13: An example of Product GUI Interface implemented by a VBA macro in CATIA V5 for the case study 
of the pipe bundle cleaner these are 15 parameters differentiated into 11 dimensional parameters (red vertices) and 4 kinematic ones (green vertices). With the aim of pointing out the impact of the parameter modification on skeleton entities and assembly components, the SSM graph visualizes the relations (or formulas) as inter or intra-plane edges between nodes. An intra-plane relation on the upper layer shows a link between two or more parameters due to a given formula. Red and green interplane relations describe respectively the path of dimensional and kinematic parameters modification on assembly members and skeleton geometric entities impacted.

To highlight better these dependencies, the skeleton and components elements impacted inherit the color of the relation type, as shown in Figure 14. The application of the SSM to the case study points out, at first glance, a design intent focused on dimensional changes in the scissor lift (left part of the bottom layer) since the other components are designed to contain standard hydraulic accessories and thus they do not need to scale with the product.

\section{Conclusion}

The skeleton, i.e. a control structure able to drive the main positions, dimensions and space allocations of the members of complex products, allows to efficiently managing the scalability and responsiveness of the product to design modifications. Recently, new methodologies have shown that the engineering knowledge coming from

\begin{tabular}{|c|l|}
\hline$O$ & PART \\
\hline$O$ & IMPACTED PART \\
\hline$\square$ & DIMENSIONAL PARAMETER \\
\hline- & DIMENSIONAL RELATION \\
\hline$O$ & SKELETON ENTITY \\
\hline$O$ & IMPACTED SKELETON ENTITY \\
\hline$\square$ & KINEMATIC PARAMETER \\
\hline- & KINEMATIC RELATION \\
\hline- & PARAMETER RELATION \\
\hline
\end{tabular}

downstream stages of the product development process can be coded and embedded in the skeleton. The SKL$A C D$ approach, implemented here through a plain case study, allows identifying a skeleton graph starting from engineering information on assembly sequence planning. This graph includes the reference geometrical entities and the mutual relationships to be embedded in the related skeleton. This approach overturns the traditional methodology where the step for assembly sequence definition follows the design detailing phase, reducing the iterations due to the definition of poor assembly requirements.

With the purpose of promoting the knowledge of skeleton-based modelling techniques, that have a great relevance for training professional, technical and mechanical engineers this paper has described the different types of skeleton implemented and the related fields of use. Particular emphasis has been placed to the need of enriching the tools currently available for the display and the management of parameters paths. This is an important issue especially with a view to better support the designer when identifying and keeping track of the parameters involved by the several formulas defined in the CAD model of a complex product. A multilayer graph representation, the SSM graph, has been proposed here as an effective tool to visualize and differentiate, at first glance, the relations, the design parameters and the impact of their modification on skeleton entities and components of the assembly. This is useful especially in the case of a topdown design approach where the reuse of parameters makes the tracing of the related paths more complex.

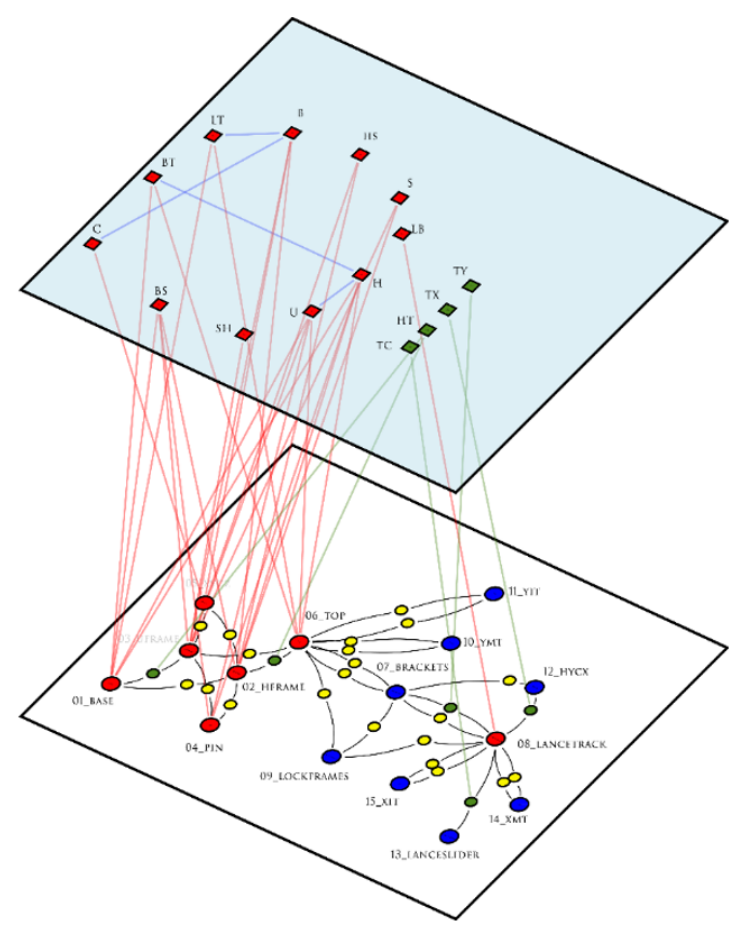

» Figure 14: The Skeleton String Model graph (SSM graph) 


\section{References}

Chandrasegaran, S. K., Ramani, K., Sriram, R. D., Horváth, I., Bernard, A., Harik, R. F. \& Gao, W. (2013) The evolution, challenges, and future of knowledge representation in product design systems. Computer-Aided Design. 45 (2), 204-228. Available from: doi: 10.1016/j.cad.2012.08.006

Demoly, F., Toussaint, L., Eynard, B., Kiritsis, D. \& Gomes, S. (2011) Geometric skeleton computation enabling concurrent product engineering and assembly sequence planning. Computer-Aided Design. 43 (12), 1654-1673. Available from: doi: 10.1016/j. cad.2011.09.006

Desrochers, A. \& Clément, A. (1994) A dimensioning and tolerancing assistance model for CAD/CAM systems. The International Journal of Advanced Manufacturing Technology. 9 (6), 352-361. Available from: doi: 10.1007/BF01748479

Gero, J. S. \& Kannengiesser, U. (2014) The function-behaviour-structure ontology of design. In: Chakrabarti, A. \& Blessing, L.T.M. (eds). An Anthology of Theories and Models of Design. London, Springer, pp. 263-283.

Marchenko, M., Behrens, B. A, Wrobel, G., Scheffler, R. \& Pleßow, M. (2011) A New Method of Visualization and Documentation of Parametric Information of 3D CAD Models. Computer-Aided Design and Applications. 8 (3), 435-448. Available from: doi: 10.3722/ cadaps.2011.435-448
McGee, F., Ghoniem, M., Melançon, G., Otjacques, B. \& Pinaud, B. (2019) The State of the Art in Multilayer Network Visualization. Computer Graphics Forum. 38 (6), 125-149. Available from: doi: 10.1111/cgf.13610

Mun, D., Hwang, J. \& Han, S. (2009) Protection of intellectual property based on a skeleton model in product design collaboration. Computer-Aided Design. 41 (9), 641-648. Available from: doi: 10.1016/j. cad.2009.04.007

Rocca, G. L. (2012) Knowledge based engineering: Between Al and CAD. Review of a language-based technology to support engineering design. Advanced Engineering Informatics. 26 (2), 159-179. Available from: doi: 10.1016/j.aei.2012.02.002

Vielhaber, M., Burr, H., Deubel, T., Christian W. \& Haasis, S. (2004) Assembly-oriented Design in Automotive Engineering. In: Majranovic, D. (Ed.) DS 32: Proceedings of DESIGN 2004, 8th INTERNATIONAL DESIGN CONFERENCE, DESIGN 2004, 18 - 21 May, Dubrovnik, Croatia. pp. 539-546.

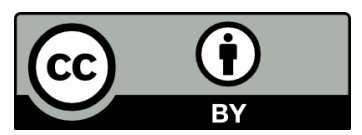

(C) 2021 Authors. Published by the University of Novi Sad, Faculty of Technical Sciences, Department of Graphic Engineering and Design. This article is an open access article distributed under the terms and conditions of the Creative Commons Attribution license 3.0 Serbia (http://creativecommons.org/licenses/by/3.0/rs/). 